\title{
A brief history of pulsar time
}

\section{R. D. Blandford}

RADIO pulsars were discovered ${ }^{1}$ because their sources, spinning neutron stars, keep time with extraordinary precision. Indeed, some rival the very best atomic clocks. But they are not perfect clocks. Allowance must be made for their steady deceleration due to the action of an electromagnetic torque; and some undergo sudden, unpredictable increases in their frequencies, known as glitches. One of the best studied pulsars is PSR0531+21, more famous as the central pulsar of the Crab Nebula - the relic of a supernova explosion observed by Chinese astronomers in AD 1054. A large glitch on 29 August 1989 shortened this pulsar's 33-millisecond period by about 3 nanoseconds, a much bigger jump than in previous glitches. On page 706 of this issue ${ }^{2}$, Lyne, Graham Smith and Pritchard present an analysis of the times of arrival of pulses from this pulsar which suggests a physical description of the interior of the neutron star that is both more complicated and more intriguing than any that has been seen so far.

By now, many pulsars have been found to undergo glitches. The most capricious example is the Vela pulsar, PSR0833-45, whose frequency has jumped by a few parts per million on eight occasions over 20 years. After each glitch, there was a temporary increase in the rate of slowing down so that the star appears to be trying to recover the frequency it would have had, had it not glitched. The best scrutinized Vela glitch, which occurred on Christmas Eve in 1988, had at least three distinguishable recovery timescales, the shortest being 10 hours and the longest being 3 months ${ }^{3}$.

However, no two glitching pulsars behave similarly. For example, PSR0355+54, which underwent the largest glitch of all in 1986, exhibited almost no recovery ${ }^{4}$. In addition, PSR1737-30, which was first found to glitch in 1987, was most prolific, performing four encores over the next two years 5 . Now we can add the Crab pulsar to this list. Its recent glitch is unique in revealing three separate recoveries, of which two are negative so that the frequency slowly increases relative to preglitch expectations. Furthermore, the steady deceleration of the Crab pulsar is apparently slightly faster now, in contrast with other glitching pulsars, which generally return to their pre-glitch slowing-down rate.

Faced with this variety, it should come as no surprise to learn that theoretical astrophysicists have had a very hard time persuading observers (and each other) that they understand glitches. Most published explanations have failed to predict the behaviour of future glitches. (One anonymous physicist appeared so confident that the first Vela glitch was a singular event that he was offered a Faustian bargain to live until the next. Fortunately, he had the wisdom to decline the offer.) As is so often the case in theoretical astrophysics, two approaches have been followed, working inductively, backwards from the data and deductively, forwards from physical theories of neutron-star matter.

The simplest, phenomenological interpretation of the results of Lyne et al. is that the neutron star contains four distinct zones just as the interior of the Earth is known to comprise a solid core, a liquid outer core, a solid mantle and a crust. In the neutron star, the four zones can spin with different frequencies, but just before the glitch, they are in synchrony. During the glitch, the star undergoes some structural change and, according to the principle of conservation of angular momentum, all four zones start spinning with different frequencies.

The pulses that we observe are generated in the magnetosphere outside the star and, in the case of the Crab pulsar, we must say that this is attached to the third-fastest component. What happens next, in this interpretation, is that frictional forces act between the different zones, rather like the clutch in a gearbox. The zone that is linked to the magnetosphere, and is responsible for the pulsar clock, is first spun up by the friction from a faster zone until both components have a common rotational frequency. Then these two components are decelerated by a slower component and, finally, the remaining, faster zone exerts a diminishing spin-up torque that leaves the components in synchrony once more. This model describes the timing record.

The alert reader might ask at this point: how can one zone be in contact with three others? After all, the liquid core of the Earth naturally has two boundaries. This brings us to the deductive model. The answer is believed to involve a remarkable property of the neutrons in the interior of the star they are thought to be superfluid ${ }^{6}$. This means that in certain density ranges, the neutrons can flow through the crystalline lattice formed by the ions with relatively little resistance, just like very cold liquid helium in the laboratory.

There is a problem, though - perfect superfluids are not supposed to rotate. The way out is that the rotation (strictly a quantity known as the vorticity) is concentrated in a bundle of parallel vortex lines (separated from each other by about 20 micrometres in the case of the Crab pulsar), whose filamentary cores contain normal, non-superfluid neutrons ${ }^{7}$. As the star slows down fewer of these vortex lines are needed and so they ought to move away from each other. However, there is a second complication. The vortex lines may get pinned by the ions in the lattice. This means that as the magnetosphere and the ions, which are linked by magnetic field, slow down, the neutron superfluid may keep on spinning with the same frequency.

When the frequency difference between the star and the lattice gets too large, the stresses in the crust become so great that something has to give and there is an exotic form of starquake. Some older (and perhaps colder) neutron stars, like PSR0355+54, may behave like this. Alternatively, after a glitch in a young (and perhaps hotter) pulsar like the Crab or Vela, the vortex lines may creep slowly through the lattice, reducing the velocity difference between the neutrons and the ions, and exerting the frictional drag that characterizes the recovery ${ }^{8}$. If this is what is happening, then in order to account for the behaviour of the Crab pulsar, some superfluid zones must be left by the glitch spinning faster than the magnetosphere.

Undoubtedly, more detailed models will accommodate the slow spin-ups in the Crab pulsar. However, as Lyne et al. remark, there is a fairly fundamental difficulty if the pulsar has undergone glitches like this one ever since its birth, as the moment of inertia of the mobile neutron superfluid would have to be as large as 5 per cent of that of the whole star, far larger than is allowed by models of neutron star interiors. Despite the observational and theoretical progress that has already been made, understanding and predicting neutron starquakes is proving to be no easier than dealing with their terrestrial counterparts.

R. D. Blandford is in the Department of Theoretical Astrophysics, California Institute of Technology, Pasadena, California 91125, USA.

\footnotetext{
1. Hewish A Bell S J Pilkington, J. D. H.. Scott, P. F. \& Collins, R. A Nature 217, $709-713$ (1968)

Collins, R. A. Nature 217, $709-713(1968)$
Lyne, A. G. Graham Smith, F. \& Pritchard. R. S. Nature 359, $706-707$ (1992)

3. Flanagan. C. S. Nature 345, 416-417 (1990)

4. Lyne. A. G. Nature 326. 569-571 (1987)

5. McKenna, J. \& Lyne, A. G. Nature 343. 349-350 (1990)

6 Baym G Pethick. C J Pines, D \& Ruderman, M Nature 224. 872-874 (1969)

7. Feynman, R. P. Statistical Mechanics: A Set of Lectures (Benjamin, Reading, 1972)

8. Alpar. M. A. Pines. D. \& Cheng. K. S. Nature 348 $707-708(1990)$
} 